\title{
Expectativas e Percepções sobre a Gerência de Configuração de Software: Um Survey
}

\author{
Maurício R. de A. Souza ${ }^{1}$, Matheus B. R. Evangelista ${ }^{1,2}$, \\ Renata T. Moreira ${ }^{1,3}$, Ana Rouiller ${ }^{1,2}$, Eduardo Figueiredo ${ }^{4}$ \\ ${ }^{1}$ SWQuality - Consultoria e Sistemas LTDA, Recife - PE \\ ${ }^{2}$ Departamento de Estatística e Informática da Universidade Federal Rural de \\ Pernambuco (DEINFO/UFRPE) - Recife - PE - Brasil \\ ${ }^{3}$ Centro de Informática da Universidade Federal de Pernambuco (CIn/UFPE) - Recife - \\ PE - Brasil \\ ${ }^{4}$ Departamento de Ciência da Computação da Universidade Federal de Minas Gerais \\ (DCC/UFMG) - Belo Horizonte - MG - Brasil \\ \{mauricio.ronny, matheusbre, anarouiller\}@gmail.com, rtmecin.ufpe.br, \\ figueiredoddcc.ufmg.br
}

\begin{abstract}
This paper describes results of the application of a survey to investigate the perceptions and expectation of Brazilian software industry professionals about Software Configuration Management (SCM). 71 responses were obtained from professionals of 50 Brazilian software organizations. Our results point the main problems observed by these professionals, before and after the adoption of SCM, their expectation related to benefits of SCM and the perceived benefits of SCM adoption. We have identified that SCM has attended the expectation of more control over changes in work products, and has overall attended to the problems related to the lack of SCM (with an average $37 \%$ rate of problems not solved).
\end{abstract}

Resumo. Este artigo descreve os resultados da aplicação de um survey para investigar as percepções e expectativas dos profissionais de software a respeito do processo de gerência de configuração de software (GCS). Foram coletados 71 respostas de profissionais de 50 organizações da indústria de software brasileira. Entre os resultados obtidos elencamos os principais problemas observados antes e depois da adoção da GCS, as expectativas dos profissionais de software em relação a processo e os beneficios observados. Identificamos que a GCS tem atendido principalmente a expectativa de maior controle sobre mudanças e que, na população analisada, a GCS tem solucionado problemas atribuidos a sua falta (com média de 37\% de problemas não solucionados).

\section{Introdução}

O papel principal da gerência de configuração é garantir que a produtividade não seja prejudicada por erros, problemas ou mudanças [Babich, 1986]. Desta forma a gerência de configuração é usualmente segmentada nas seguintes atividades [IEEE, 2004; ISO/IEC, 2008; SOFTEX, 2012]: identificação da configuração, controle da configuração, contabilização da situação da configuração, avaliação e revisão da configuração e gerenciamento de liberação e entrega. 
A gerência de configuração não é uma área exclusiva ao contexto de software, e, quando aplicada a este fim, possui desafios particulares. Devido a natureza abstrata e maleável e sua constante necessidade de adaptação ao ambiente, mudanças são intrínsecas ao software [DART, 1991]. Estas mudanças podem gerar falhas difíceis de serem rastreadas, devido à dependências não evidentes entre os componentes. Além disso, a atividade de desenvolvimento de software é primariamente intelectual e orientada ao trabalho em equipe, o que faz com que a Gerência de Configuração de Software (GCS) tenha um papel importante na gestão, a fim de minimizar o retrabalho, conflitos e perda de informação.

Diferentes contextos implicam em diferentes necessidades relacionadas a GCS. Conforme dados da ABES - Associação Brasileira das Empresas de Software [ABES, 2013], o mercado de software brasileiro é explorado por cerca de 10.700 empresas, dedicadas ao desenvolvimento, produção, distribuição de software e de prestação de serviços. Daquelas que atuam no desenvolvimento e produção de software, cerca de $93 \%$ são classificadas como micro e pequenas empresas. Dependendo do contexto da organização, a gerência de configuração pode estar relacionada a diferentes problemas e expectativas dos profissionais da área.

Este artigo apresenta uma investigação sobre a percepção dos profissionais do mercado de software brasileiro sobre a GCS. Utilizou-se uma pesquisa de opinião para tentar identificar quais as principais expectativas mantidas por esses profissionais a respeito do processo e quais os reais benefícios observados com a sua institucionalização.

As contribuições esperadas com esta pesquisa envolvem a atualização da percepção sobre os problemas relacionados a GCS e o entendimento das necessidades e expectativas dos profissionais. A partir do entendimento dos problemas identificados, ações poderão ser propostas para que a implantação da GCS em organizações de software atenda melhor às expectativas apontadas pelos profissionais da indústria.

Além desta seção introdutória, este artigo está organizado conforme a seguinte configuração de seções: A Seção 2 apresenta trabalhos relacionados. A Seção 3 descreve a metodologia de pesquisa. Na Seção 4 são apresentados os resultados obtidos. A Seção 5 mostra as ameaças a validade. Na Seção 6 são feitas as considerações finais do trabalho e na seção 7 são descritos os agradecimentos.

\section{Trabalhos relacionados}

A GCS é um processo recorrente em padrões e normas de qualidade [IEEE, 2004; ISO/IEC, 2008; SEI, 2010; SOFTEX, 2012]. Vários estudos apontam que organizações têm investido recursos nesta área de conhecimento e que a GCS e a gestão de solicitações de mudança estão entre os processos de maior interesse para a melho ria em organizações de software [Pino et al., 2007].

Dois estudos concentraram esforços em avaliar e entender o processo de gerência de configuração em relação aos problemas, expectativas e benefícios na prática [Aberdeen Group, 2007; Forrester Consulting, 2006]. Em 2007, um relatório técnico consolidando medidas de referência (benchmark) sobre a gerência de configuração (GC) foi produzido [Aberdeen Group, 2007]. A partir da aplicação de um questionário foram levantados os desafios para gerenciar efetivamente a configuração de produtos, a efetividade de uma variedade de procedimentos e infraestruturas de gerência de 
configuração existentes, o uso de automação para apoiar e promover a visibilidade da informação e os resultados e benefícios de abordagens tecnológicas, processuais e organizacionais para melhoria da gerência de configuração de software. A pesquisa foi realizada sobre uma população de mais de 200 empresas de engenharia, tecnologia da informação e software, engenharia aeroespacial, indústria, finanças, logística, e outras. Entre os resultados do relatório, cita-se [Aberdeen Group, 2007]:

- Pressões que motivam a melhoria da GC: Melhoria de qualidade de produtos (41\%), melhoria no tempo de entrega (time to market) (33\%), redução de custos de desenvolvimento (29\%), redução de custos do ciclo de vida do produto $(28 \%)$ e redução de custos de produção $(27 \%)$;

- Principais desafios para a GC efetiva: Saber com precisão da situação da configuração (componentes e compatibilidade ao longo das versões) (40\%), gerenciar mudanças ao longo de configurações (37\%); manter sincronia entre a situação da configuração e mudanças $(31 \%)$, gerenciar situação e mudanças em variações da configuração (29\%);

- Responsabilidades sobre a GC: a maior parte das organizações consideradas "Best in Class", pelo estudo, têm uma figura gerencial à frente dos processos de gerência de configuração (57\%) e um nível alto de controle centralizado sobre os processos de gerência de configuração e mudança (33\%), ou processos de aprovação e revisão entre departamentos (47\%).

Outro estudo de survey sobre gerência de configuração [Forrester Consulting, 2006] levantou os principais problemas relacionados a falta de processos de gerência de mudanças. Como resultado apontou os seguintes problemas: Baixa qualidade do software $(75 \%)$, insatis fação de clientes $(72 \%)$, retrabalho desnecessário $(71 \%)$, prazos perdidos (68\%), altos custos $(61 \%)$, mudanças sem sucesso $(57 \%)$ e tempo de parada em produção $(50 \%)$.

Tomando como referência os problemas e benefícios associados à gerência de configuração dos estudos acima citados, este artigo procura contribuir com uma análise sob a perspectiva dos profissionais de software da indústria nacional.

\section{Metodologia}

Segundo Easterbrook et al. (2008), uma pesquisa de survey é utilizada para identificar características de uma ampla população de indivíduos, comumente associada ao uso de questionários para a coleta dos dados. No entanto, o survey está inserido em um contexto maior [Pfleeger and Kitchenham, 2001], sendo um instrumento para coletar informações para descrever, comparar ou explicar conhecimentos, atitudes e comportamentos.

A construção e aplicação deste survey adotou o processo descrito em [Pfleeger and Kitchenham, 2001], garantindo que seja (i) resistente ao viés, (ii) apropriado e (iii) custo eficiente. Para atender aos requisitos (i) e (ii) este survey foi planejado para ser avaliado por pesquisadores e profissionais da indústria. Optou-se por construir um questionário com questões de múltiplas escolhas a fim de tornar o tempo de resposta mais eficiente (iii).

Os objetivos, construção do questionário, população alvo, amostra e respostas são descritas nas subseções a seguir. 


\subsection{Objetivos da pesquisa}

O objetivo principal da pesquisa é investigar as impressões dos profissionais da indústria de software em relação ao processo de gerência de configuração. Este objetivo deu origem às seguintes questões de pesquisa:

- QP1: Quais os principais problemas relacionados a GCS? - A partir do levantamento de problemas já descritos na literatura técnica, esta questão busca trazer um entendimento sobre quais os problemas relacionados a falta de um processo de gerência de configuração, e, quais os problemas recorrentes mesmo após a implantação de um processo de GCS.

- QP2: Quais os benefícios esperados da implantação de um processos de GCS? - Esta questão têm o objetivo de investigar o que os profissionais de software apontam como expectativas em relação a adoção ou melhoria de práticas de GCS em suas organizações, e, identificar qual a percepção destes a respeito dos benefícios que realmente foram observados com a adoção da GCS.

- QP3: Qual a influência da melhoria de processo sobre a GCS nas organizações? - Uma vez que a GCS está largamente presente em programas, modelos e normas para a melhoria de processo de software, esta questão investiga qual a influência (positiva, negativa ou inexistente) da adoção destes padrões em relação às atividades de GCS nas organizações de software.

Diferentes perspectivas em relação à características da organização, de seus profissionais e de seus processos foram consideradas ao avaliar os resultados para as questões de pesquisa estabelecidas, conforme descrito nas seções seguintes.

Para esta pesquisa não há hipóteses a serem validadas, uma vez que o estudo tem caráter investigativo/exploratório. O objetivo desta pesquisa está limitado às impressões dos profissionais sobre os problemas, atividades e benefícios relacionados a gerência de configuração de suas respectivas organizações. Não é objetivo desta pesquisa avaliar ou comparar organizações, ou mesmo, avaliar a efetividade do processo de gerência de configuração.

\subsection{Questionário}

A fim de responder às questões de pesquisa, considerando as necessidades de informação para caracterização dos participantes, um questionário com 16 perguntas foi definido. $\mathrm{O}$ questionário foi composto por perguntas diretamente ligadas às questões de pesquisa e perguntas para investigar características das organizações, dos seus processos de GCS e da forma de atuação dos entrevistados nestas organizações. As questões definidas são apresentadas no Quadro 1.

O questionário foi desenvolvido em iterações nas quais versões candidatas eram construídas e aplicadas em um grupo de controle. O grupo de controle foi formado por pesquisadores e profissionais da indústria e teve como objetivo a análise das perguntas e opções de respostas, para avaliação de clareza e consistência do questionário. A versão final do questionário foi construída e publicada somente após a aprovação pelo grupo de controle. Este ciclo foi importante para evitar viés, conforme os requisitos básicos de um survey [Pfleeger and Kitchenham, 2001]. 
Quadro 1. Questionário

\begin{tabular}{|c|c|}
\hline ID & Questões e Respostas \\
\hline Q1 & Identificação do entrevista do (nome, organização, cargo) \\
\hline Q2 & Há quanto tempo você atua na organização? \\
\hline Q3 & Que tipo de atividades caracterizam a organização onde atua? \\
\hline Q4 & Qual o tamanho da organização? \\
\hline Q5 & $\begin{array}{l}\text { A organização já foi certificada em algum programa, modelo ou norma de qualidade? (ou } \\
\text { está realizando um programa de melhoria baseado em algum modelo) }\end{array}$ \\
\hline Q6 & $\begin{array}{l}\text { Que tipo de perfil melhor caracteriza as atividades que você desempenha na organização } \\
\text { onde atua? }\end{array}$ \\
\hline Q7 & Como você avalia seu grau de conhecimento/experiência em gerência de configuração? \\
\hline Q8 & $\begin{array}{l}\text { Como você interage com as atividades de gerência de configuração na organização onde } \\
\text { atua? }\end{array}$ \\
\hline Q9 & Avalie a situação das atividades de gerência de configuração na organizaçã o onde atua. \\
\hline Q10 & $\begin{array}{l}\text { Há quanto tempo a organização mantém o processo/atividades de gerência de } \\
\text { configuração? }\end{array}$ \\
\hline Q11 & $\begin{array}{l}\text { Identifique os problemas mais relevantes que eram parte da realidade da organização } \\
\text { ANTES da gerência de configuração (ou problemas atuais, caso a organização não realize } \\
\text { atividades de gerência de configuração) }\end{array}$ \\
\hline Q12 & $\begin{array}{l}\text { Qual é ou foi a sua principal expectativa em relação a implantação da gerência de } \\
\text { configuração de software na organização onde atua? }\end{array}$ \\
\hline Q13 & $\begin{array}{l}\text { Quais os principais benefícios observados após a institucionalização de práticas (ou } \\
\text { melhorias) de gerência de configuração? }\end{array}$ \\
\hline Q14 & $\begin{array}{l}\text { Quais problemas relacionados a gerência de configuração persistem (ou surgiram) na } \\
\text { organização? }\end{array}$ \\
\hline Q15 & $\begin{array}{l}\text { Em uma escala de um a cinco, como você classifica a importância do processo de gerência } \\
\text { de configuração dentro da organização? }\end{array}$ \\
\hline Q16 & $\begin{array}{l}\text { Caso a organização tenha sido submetida a (ou esteja conduzindo) um programa de } \\
\text { melhoria de processo, como isto influenciou no processo/atividades de gerência de } \\
\text { configuração? }\end{array}$ \\
\hline
\end{tabular}

\subsection{População, a mostras e respostas}

A pesquisa teve como alvo profissionais atuantes em organizações que desempenham atividades relacionadas a software (fábrica, manutenção e evolução de produtos $\mathrm{e}$ prestação de serviços). A pesquisa foi divulgada em redes sociais, através de e-mail em listas de discussão e redes de contatos profissionais dos pesquisadores envolvidos. A publicação do survey e coleta das respostas foi gerenciada através da ferramenta Murvey [Murvey, 2014]. O questionário permaneceu online entre $1^{\circ}$ de Março à $1^{\circ}$ de Abril de 2014 e foi respondido por um total de 71 profissionais de 50 organizações distintas.

Dos profissionais participantes da pesquisa que responderam a pergunta Q2, 18 (25\%) têm menos de 1 ano de atuação na organização atual, 28 (39\%) têm entre 1 a 3 anos, $11(15 \%)$ têm entre 3 a 5 anos, $9(13 \%)$ têm entre 5 e 10 anos e $5(7 \%)$ têm mais 
de 10 anos. Em relação a auto avaliação sobre o grau de conhecimento ou experiência com atividades de gerência de configuração (Q7), numa escala de 1 (muito baixo) à 5 (muito alto) os participantes se enquadraram na média de 3,19 (Razoável). Destes, 13 (18\%) são responsáveis pelo processo de GCS das organizações onde atuam, $36(50 \%)$ realizam algumas atividades de GCS, 13 (18\%) apenas solicitam ações de GCS, e 9 (13\%) não interagem com o processo de GCS da organização (Q8).

Os participantes foram questionados quanto ao tipo de atividades que executavam em suas organizações (Q6). 3 participantes (4\%) (alegaram desempenhar atividades a nível estratégico (direção, gestão executiva, alinhamento estratégico, gestão de portfólios). 15 participantes (21\%) alegaram desempenhar atividades a nível tático (gestão de projetos, programas e equipes). 43 participantes (61\%) alegaram desempenhar atividades a nível operacional (desenvolvimento, operações, atendimento). Por fim, 10 participantes (14\%) alegaram desempenhar atividades a nível apoio (controle de processos, auditorias e atividades de apoio a atividade fim da organização).

Quanto ao perfil das organizações participantes da pesquisa, identificamos:

- Tamanho: $24 \%$ são micro empresas (até 9 funcionários), 38\% são de pequeno porte (de 10 a 49 funcionários), 10\% são de médio porte (50 a 99 funcionários) e 28\% são de grande porte (a partir de 100 funcionários);

- Tipo de Atividades: Podendo se enquadrar em mais de uma opção, 35 empresas realizam atividades de manutenção e evolução de produtos; 30 realizam atividades de fábrica de software; 24 realizam atividades de prestação de serviços de software e 6 apontaram que realizam outros tipos de atividades.

- Certificações e Programas de Melhoria de Processo: 22 organizações (44\%) participaram ou estão envolvidas em programas de melhoria de processo ou ações de certificação;

Por motivos de confidencialidade, nomes de pessoas e organizações serão omitidos na apresentação dos resultados da pesquisa.

\section{Resultados}

Nesta seção serão apresentados os resultados obtidos da execução e análise dos questionários.

\subsection{Principais problemas relacionados a GCS (QP1)}

Foram definidos seis problemas (Quadro 2) inspirados no que estudos anteriores apontam sobre a carência de um processo de GCS em organizações de software [Aberdeen Group, 2007; Forrester Consulting, 2006]. A partir destes problemas, foi questionado (Q11 e Q14) aos participantes quais eram os mais relevantes e quais faziam parte da realidade de suas respectivas organizações em dois momentos: (i) antes e (ii) depo is da implantação de um processo ou atividades de GCS (múltiplas repostas eram permitidas).

Em ambas as situações (i) e (ii), o problema mais recorrente foi a dificuldade em controlar e rastrear mudanças e impactos (P6), o que a literatura técnica normalmente atribui à atividade de Controle de Mudanças da GCS [ISO/IEC, 2008; SEI, 2010; SOFTEX, 2012]. O problema menos citado em (i) e (ii) foi $\mathrm{P} 4$, referente a releases com 
falhas ou incompletas. O problema em que houve maior diferença entre (i) e (ii) foi P2 perda de dados e informações. Os resultados são apresentados na Tabela 1.

Quadro 2. Problemas relacionados a GS

\begin{tabular}{l|l}
\hline ID & Problema \\
\hline P1 & Dificu ldade de mapear versões \\
P2 & Perda de dados e informações \\
P3 & Retrabalho \\
P4 & Releases com falhas ou inco mpletas \\
P5 & Dificuldade em gerenciar o trabalho em equipe \\
P6 & Dificuldade em controlar e rastrear mudanças e impactos \\
P7 & Outros \\
\hline
\end{tabular}

Tabela 1. Principais problemas relacionados a GCS

\begin{tabular}{c|cc}
\hline Problema & $\begin{array}{c}\text { Totale } \% \\
\text { Ocorrência(i) }\end{array}$ & $\begin{array}{c}\text { Totale \% } \\
\text { Ocorrência(ii) }\end{array}$ \\
\hline P1 & $34(47,9 \%)$ & $14(19,7 \%)$ \\
P2 & $34(47,9 \%)$ & $15(21,1 \%)$ \\
P3 & $32(45,1 \%)$ & $14(19,7 \%)$ \\
P4 & $27(38,0 \%)$ & $13(18,3 \%)$ \\
P5 & $33(46,5 \%)$ & $14(19,7 \%)$ \\
P6 & $41(57,7 \%)$ & $27(38,0 \%)$ \\
P7 & $4(5,6 \%)$ & $4(5,6 \%)$ \\
\hline
\end{tabular}

Ao analisar situações em que um participante apontou um mesmo problema na situação (i) e (ii), foi levantada a taxa de reincidência observada para cada problema (Tabela 2). Foi observado uma taxa média de $36,9 \%$ de reincidência dos problemas, conforme a percepção dos participantes. Isto implica que $36,9 \%$ dos entrevistados que identificaram um determinado problema na organização, não perceberam resolução com a inserção de práticas de GCS. Neste cenário, o problema individual que teve a maior taxa de reincidência foi também P6 (46,3\%), sendo pertinente maior investigação sobre o fato.

Quando analisamos sob diferentes perspectivas, identificamos os seguintes pontos:

- Tipo de organização: Em relação às organizações com diferentes atividades fim, os resultados diferem ligeiramente da organizações de manutenção de software, onde o problema P1 apresenta um aumento de ocorrência;

- Perfil dos profissionais: Para profissionais cujo nível de atividade é o operacional, o problema P1 foi o mais recorrente em (i) $(51,2 \%)$, seguido de P6 e P5 (48,8\%). Em (ii), P6 permanece como o problema mais recorrente. A média da taxa de reincidência de problemas aumenta, nesta amostra da população, para $48,4 \%$. 
Tabela 2. Taxa de recorrência de problemas

\begin{tabular}{c|ccc}
\hline Problema & $\begin{array}{c}\text { Problema } \\
\text { Identificado em (i) }\end{array}$ & $\begin{array}{c}\text { Problema Identificado } \\
\text { em (i) e (ii) }\end{array}$ & \% Reincidência \\
\hline P1 & 34 & 11 & $32,4 \%$ \\
P2 & 34 & 13 & $38,2 \%$ \\
P3 & 32 & 11 & $34,4 \%$ \\
P4 & 27 & 10 & $37,0 \%$ \\
P5 & 33 & 11 & $33,3 \%$ \\
P6 & 41 & 19 & $46,3 \%$ \\
\hline Média & & & $36,9 \%$ \\
\hline
\end{tabular}

\subsection{Expectativas e benefícios relacionados a GCS (QP2)}

Para avaliar quais eram as principais expectativas dos participantes em relação aos benefícios esperados da implantação de um processo ou atividades de GCS, definimos uma lista de beneficios (Quadro 3) tipicamente relacionados a GCS [Amberdeen, 2007].

Quadro 3. Benefícios relacionados a GCS

\begin{tabular}{|c|c|}
\hline ID & Expectativas/Benefícios \\
\hline B1 & Melhoria da Qualidade de produtos e processos. \\
\hline B2 & $\begin{array}{l}\text { Garantir controle mais eficiente sobre mudanças realizadas } \\
\text { em produtos de trabalho. }\end{array}$ \\
\hline B3 & $\begin{array}{l}\text { Controle mais efetivo sobre a situação dos produtos de } \\
\text { trabalho. }\end{array}$ \\
\hline B4 & Diminuição de custos. \\
\hline B5 & Facilitar o trabal ho e a comuni cação em equipe. \\
\hline B6 & Diminuição do Tempo de en trega \\
\hline
\end{tabular}

Sobre este aspecto, formulamos duas questões. A primeira, em Q12, sobre (i) qual a principal expectativa de benefício do entrevistado da implantação da GCS (apenas uma alternativa permitida), e, a segunda, em Q13, sobre (ii) quais beneficios foram realmente percebidos com a implantação de GCS (múltiplas alternativas permitidas). Na Tabela 3 são apresentados os resultados destas questões.

Os benefícios mais recorrentes enquanto expectativas (i) foram B2 e B1. Quanto ao resultado de B1, era esperado este resultado conforme [Aberdeen Group, 2007] e [Forrester Consulting, 2006], em que a qualidade de produtos é um ponto chave relacionado a melhoria do processo de GCS. No entanto, em divergência com estes estudos, B4 e B6 tiveram os menores resultados enquanto expectativas relatadas pelos participantes. Uma possível justificativa para isto é a participação majoritária de entrevistados que desempenham atividades de nível operacional (61\%), 
Tabela 3. Resultado dos benefícios relacionados a GCS

\begin{tabular}{c|cc}
\hline $\begin{array}{c}\text { Expectativas/ } \\
\text { Benefícios }\end{array}$ & $\begin{array}{c}\text { Totale } \% \\
\text { Expectativa(i) }\end{array}$ & $\begin{array}{c}\text { Totale } \% \\
\text { Benefício } \\
\text { Observado (ii) }\end{array}$ \\
\hline B1 & $23(32,4 \%)$ & $26(36,6 \%)$ \\
B2 & $26(36,6 \%)$ & $43(60,6 \%)$ \\
B3 & $9(12,7 \%)$ & $34(47,9 \%)$ \\
B4 & $1(1,4 \%)$ & $5(7 \%)$ \\
B5 & $9(12,7 \%)$ & $29(40,8 \%)$ \\
B6 & $1(1,4 \%)$ & $12(16,9 \%)$ \\
Outros & $2(2,8 \%)$ & $1(1,4 \%)$ \\
Nenhum & $0 \%$ & 0 \\
\hline
\end{tabular}

Ao analisarmos os resultados de (ii), B2 é novamente o resultado mais observado. No entanto, B3 (controle sobre a situação de produtos de trabalho) foi o segundo maior resultado e B5 (facilitação da comunicação e trabalho em equipe) o terceiro. Muito embora os resultados de B3 e B5 tenham sido baixos em (i), observa-se que estes benefícios são amplamente notados após a implantação da melhoria do processo de GCS (ii).

Isolando os resultados dos participantes que apontaram os mesmos beneficios em (i) e (ii), ou seja, os participantes que observaram resultados para sua principal expectativa, identificamos os resultados apresentados na Tabela 4.

Os benefícios B3 e B2 foram os que representaram maior taxa de satisfação às expectativas, com $78 \%$ e $77 \%$, respectivamente. Para B1, que foi o segundo fator mais citado enquanto expectativa (i), teve uma taxa de satisfação de $57 \%$. É relevante a investigação sobre os fatores que influenciam os resultados positivos de B2 e B1. As expectativas B4 e B6 apresentaram taxa de satisfação de $0 \%$ devido ao baixo número de participantes que as selecionaram como principal expectativa (apenas um em cada).

Tabela 4. Taxa de Satisfação às Expectativas

\begin{tabular}{c|ccr}
\hline Expectativas & $\begin{array}{c}\text { Benefícios } \\
\text { em (i) }\end{array}$ & $\begin{array}{c}\text { Benefícios em } \\
\text { (i) e (ii) }\end{array}$ & \% Satisfação \\
\hline B1 & 23 & 13 & $57 \%$ \\
B2 & 26 & 20 & $77 \%$ \\
B3 & 9 & 7 & $78 \%$ \\
B4 & 1 & 0 & $0 \%$ \\
B5 & 9 & 5 & $56 \%$ \\
B6 & 1 & 0 & $0 \%$ \\
\hline
\end{tabular}

\subsection{Melhoria de processo de softwa re $x$ GCS (QP3)}

A respeito da influência de programas de melhoria de processo de software (MPS) sobre o processo de gerência de configuração das organizações, $58 \%$ das respostas apontaram que houve melhorias consideráveis nos seus processos de gerência de configuração, 
$18 \%$ alegaram que trouxe pequenas melhorias, 5\% apontaram que o processo de gerência de configuração só foi introduzido por conta de programas melhoria e 5\% apontaram que programas de melhoria não causaram influências sobre seus processos de gerência de configuração (Figura 1). Os percentuais apresentados na figura são referentes aos 43 profissionais $(60 \%$ da amostra total) atuantes nas 22 organizações que participaram ou estão participando de programas de melhoria.

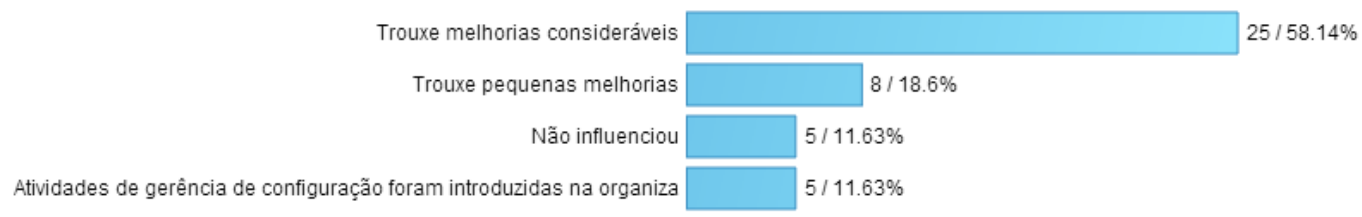

Figura 1. Impacto da MPS sobre atividades de GCS

\subsection{Outras análise}

A seguir serão apresentadas outras facetas observadas durante a aplicação do survey.

\subsubsection{Grau de institucionalização do processo de GCS}

Ao analisar a percepção dos profissionais a respeito da situação das atividades de sua organização (Q9) foi identificado que: 20 participantes (28\%) apontaram que suas organizações possuem um processo definido (planejado) para GCS e institucionalizado (executado e mantido); 16 participantes $(23 \%)$ apontaram que algumas atividades de GCS foram padronizadas independente de um processo definido; 19 participantes (27\%) apontaram que algumas atividades de GCS são realizadas, porém não há um padrão e dependem de iniciativas individuais; 13 participantes (18\%) apontaram que há um processo definido porém não plenamente executado; 03 participantes $(4 \%)$ apontaram que não são realizadas atividades de GCS.

Das organizações em que há um processo definido e institucionalizado, $25 \%$ são de grande porte, $15 \%$ de médio porte, $40 \%$ de pequeno porte e $20 \%$ são micro. Considerando apenas este grupo, observamos que $75 \%$ apontaram que suas organizações participaram em programas de melhoria de processo.

Dos profissionais inseridos neste contexto, $15 \%$ atuam no nível de apoio organizacional, $25 \%$ no nível tático e $60 \%$ no nível operacional. Sob a perspectiva destes profissionais, observamos:

- Para a área de apoio, a implantação do processo de GCS reduziu em 50\% P5 e aumentou em $50 \%$ P3 e os outros problemas não perceberam impacto;

- Para área de apoio, os principais benefícios que o processo de GCS trouxe para a empresa foram: B2, B1, B3 e B5, com $67 \%, 33 \%, 33 \%, 33 \%$ respectivamente.

- Para o nível tático, o processo de GCS resolveu 100\% os problemas com P1 e $\mathrm{P} 2$, reduziu em $50 \%$ os problemas com $\mathrm{P} 5$, P4 e P3, e, reduziu em $33 \%$ o problema com $\mathrm{P} 6$.

- Os benefícios percebidos pelo nível tático após a implantação de GCS foram: B1, B2 e B5 com $80 \%, 80 \%$ e $20 \%$, respectivamente.

- Para o nível ope racional o processo de GCS resolveu em $40 \%$ o problema de $\mathrm{P} 1, \mathrm{P} 2$ e $\mathrm{P} 6,32 \%$ de $\mathrm{P} 3$ e P4 e $24 \%$ de P5. 
- Os principais benefícios percebidos com a implantação de GCS para o nível operacional foram: B2, B3, B1, B5, B6, B4, com 83\%, 83\%, 50\%, 50\%, 25\% e $17 \%$, respectivamente.

\subsubsection{Respons abilidade sobre o processo de GCS}

Ao analisar as resposta de Q9, identificamos que $36(51 \%)$ dos profissionais entrevistados realizam algumas atividades de GCS, 13 (18\%) são responsáveis pelo processo, 13 (18\%) apenas solicitam atividades de GCS e $9(13 \%)$ alegam não interagir com GCS.

Entre os profissionais que responderam que são responsáveis pelas atividades ou processo de GCS da organização, 8\% atuam no nível de apoio (um analista de teste), $69 \%$ no nível operacional (cinco desenvolvedores/analistas/programadores, um arquiteto de TI e um Scrum Master que atua também como gerente de configuração) e $23 \%$ no nível tático (dois gerentes de projetos e um gerente de configuração).

\subsubsection{Importância da GCS}

Em Q15, foi questionado o grau de importância atribuída a GCS. O resultado pode ser observado na Figura 2 e é predominantemente alta.

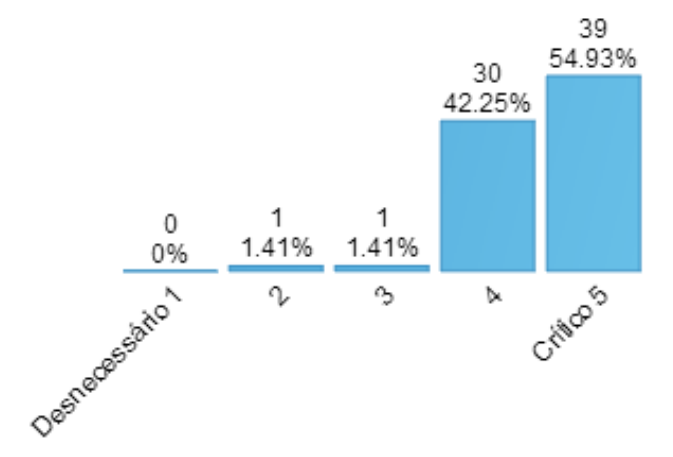

Figura 2. Avaliação da importância da GCS

\section{Ameaças a validade}

Conforme Kitchenham e Pfleger (2008), pesquisas baseadas em questionários não é um método de pesquisa simples. É necessário tempo e esforço para entender a base metodológica, bem como para criar, validar e administrar o questionário enquanto ferramenta. Para definir as ameaças à validade deste estudo, foi considerada a proposta de pesquisa de Runeson e Höst (2007) e as considerações de Pfleeger e Kitchenham (2001):

- Validade do Conteúdo: Neste caso foram avaliadas e tomadas como base pesquisas de questionário executadas anteriormente [Aberdeen Group 2007; Forrester Consulting 2006], no contexto da GCS na indústria, dos quais foram extraídas informações a respeito dos principais problemas relacionados.

- Validade da Construção: A fim de garantir que as questões definidas fossem apropriadas para a pesquisa, conforme descrito na Seção 3.2, um piloto foi executado junto a um grupo de controle formado por representantes do público alvo (profissionais da indústria) e especialistas (pesquisadores e consultores em 
melhoria de processo). O questionário foi refinado a partir de iterações de considerações do grupo de controle para garantir o máximo de adequação, clareza e suficiência das questões para a pesquisa;

- Valida de da Confiabilidade: Para garantir o máximo de confiabilidade e evitar confusão e dúvidas dos participantes em relação às questões, o questionário foi predominantemente construído com questões objetivas, com múltiplas alternativas acompanhadas por exemplos descritivos. Adicionalmente, foi permitido a participação de mais de um profissional por organização, de forma que pudéssemos verificar se as informações submetidas eram convergentes (quando não se tratava de opiniõ es pessoais);

- Viés da Amostra: O convite para participação ao questionário foi enviado por vários canais (correio eletrônico, redes sociais e abordagens diretas). Um número de indivíduos respondeu negativamente a solicitação com a justificativa de não terem conhecimento suficiente para comentar sobre a GCS em suas organizações. Além disso, foi definida uma questão (Q7) para que o participante fizesse uma auto avaliação sobre seu conhecimento no assunto, com o intuito de filtrar respostas de menor confiabilidade. No entanto, identificamos a média de 3,19 (numa escala de 1 (muito baixo) à 5 (muito alto)) na questão Q7, com desvio padrão de 0,7 , dentro da margem aceitável para a pesquisa. Quanto ao tamanho da amostra, a dificuldade de captar um número relevante de respostas neste tipo de pesquisa já foi discutido por Easterbrook et. al. (2008). Neste trabalho, foram obtidas 71 respostas, número razoá vel para a pesquisa realizada.

\section{Conclusão}

Este artigo apresentou os resultados obtidos a partir de uma pesquisa de opinião (survey) para investigar as impressões e expectativas dos profissionais da indústria de software nacional sobre o processo de gerência de configuração de software. A pesquisa foi conduzida com a meta de levantar informações para as seguintes questões:

- QP1: Quais os principais problemas relacionados a GCS? - Os resultados mostram que o problema relacionado à falta de processo de GCS mais apontado é a dificuldade de controlar e rastrear mudanças e impactos $(57,7 \%)$ seguido, em ordem de ocorrência, por dificuldade de mapear versões (47,9\%), perda de dados e informações $(47,9 \%)$, dificuldade em gerenciar o trabalho em equipe $(46,5 \%)$ e releases com falhas ou incompletas (38\%). Identificamos uma taxa média de $36,9 \%$ de persistência destes problemas após a adoção de atividades de GCS;

- QP2: Quais benefícios esperados da implantação de um processos de GCS? - Identificamos que a principal expectativa dos participantes desta pesquisa em relação ao processo de GCS residia em garantir controle mais eficiente sobre mudanças realizadas em produtos de trabalho $(36,6 \%)$ e a melhoria da qualidade de produtos e processos (32,4\%). Em relação aos benefícios observados após a adoção de atividades de GCS, foram observados: controle mais eficiente sobre mudanças realizadas em produtos de trabalho $(60,6 \%)$, controle mais efetivo sobre a situação dos produtos de trabalho (47,9\%), diminuição de custos $(40,8 \%)$ e a melhoria da qualidade de produtos e processos $(36,6 \%)$. Em relação a taxa de satisfação das expectativas apontadas, identificamos que a GCS tem atendido a expectativa de maior controle sobre mudanças $(77 \%)$, no entanto não tem 
atendido satisfatoriamente a expectativa de melhoria da qualidade de produtos e processos (57\%);

- QP3: Qual a influência da melhoria de processo sobre a GCS nas organizações? - Identificamos que $76 \%$ dos entrevistados, cujas organizações participaram de programas de melhoria, perceberam alguma melhoria em suas atividades de GCS, 5\% não observaram influências sobre suas atividades de CGS, e, 5\% alegaram que atividades de GCS só foram introduzidas em suas organizações em decorrência de programas de melhoria.

Assim, entendemos que a gerência de configuração tem resolvido parcialmente os problemas aos quais está relacionada, ainda que haja uma taxa média de $36,9 \%$ de reincidência dos problemas, conforme a percepção dos participantes da pesquisa. Investigações sobre o cenário das organizações em que os problemas persistem e nas organizações em que os problemas são resolvidos com a inserção de atividades de GCS podem ajudar a entender problemas, desafios e lições aprendidas para tratá-los.

Quanto aos benefícios esperados e observados, a GCS tem atendido a necessidade de controle sobre mudanças (B2), com satisfação em $77 \%$ dos casos, e, benefício observado por $60 \%$ dos entrevistados. No entanto, o benefício de melhoria da qualidade de produtos e processos (B1) foi observada por apenas 36\% dos entrevistados. Para os participantes que apontaram este benefício como principal expectativa, apenas $57 \%$ dos entrevistados tiveram sua expectativa atendida. Apesar de baixas expectativas em relação à melhoria da comunicação e do trabalho em equipe (B5) e o maior controle sobre a situação dos produtos de trabalho (B3), estes foram benefícios recorrentemente observados (48\% e $41 \%$, respectivamente), indicando consequências positivas do processo, mesmo que não sejam um fator primário na motivação para a adoção da GCS.

Outras observações realizadas em relação a amostra examinada são:

- Devido ao baixo número de participantes atuantes no nível Estratégico (4\%), os resultados desta pesquisa podem não corresponder às percepções deste público em relação ao processo de GCS;

- O estabelecimento de processos para a GCS estão fortemente ligados à participação das organizações em programas de melhoria de processo;

- A responsabilidade pela gerência de configuração está principalmente atribuída aos profissionais que atuam em nível operacional (desenvolvedores principalmente);

- Em geral, a população entrevistada atribuiu alta importância às atividades de GCS em suas organizaçõ es;

Como próximas ações, planeja-se a entrevistar uma amostra da população de participantes da pesquisa para obter maiores esclarecimentos sobre as respostas, além de analisar e validar os resultados apresentados. Também planeja-se uma nova aplicação do mesmo experimento em uma população em que todas as organizações tenham sido avaliadas oficialmente em modelos de qualidade para a melhoria de processo para avaliar os impactos em relação as questões QP1 e QP2.

Entre as lições aprendidas deste estudo, destacamos o valor observado do uso de questões para investigar características das organizações, a forma de atuação dos 
profissionais e o processo. Estas características permitiram que várias perspectivas sobre um mesmo dado fossem analisadas.

\section{Agradecimentos}

Este trabalho recebeu recursos financeiros da FAPEMIG (Projeto APQ-02532-12) e CNPq (Projeto 485907/2013-5).

\section{Referências}

Aberdeen Group (2007). "The Configuration Management Benchmark Report". Disponível em http://aberdeen.com/. Último acesso em 20 de abril de 2014.

ABES - ASSOCIAÇÃO BRASILEIRA DE EMPRESAS DE SOFTWARE (2013). "MERCADO BRASILEIRO DE SOFTWARE - Panoramas e Tendências 2013". . http://central.abessoftware.com.br/. Último acesso em 20 de abril de 2014.

Babich, W. A. (1986). "Software Configuration Management - Coordination For Team Productivity". Addison-Wesley (Reading, Mass.), 1986.

Dart, S. (2008). "Concepts in Configuration Management Systems". In: International Workshop On Software Configuration Management (SCM), 1991. Proceedings... Trondheim, Norway, June, p. 1-18, 1991.

Easterbrook, S. et al. (2008). "Selecting Empirical Methods for Software Engineering Research". In: SHULL, F.; SINGER, J.; S JøBERG, D. I. K. Guide to advanced empirical software engineering. [S.1.]: Springer-Verlag. p. 285-311. London, 2008.

Forrester Consulting (2006). "The Challenges Of Software Change Management" In Today's Siloed IT Organizations A Commissioned Study Conducted By Forrester Consulting On Behalf Of Serena Software. US A, 2006.

IEEE (2004). "SWEBOK - Guide to the Software Engineering Body of Knowledge, 2004 Version", IEEE Computer Society Professional Practices Committee, Los Alamitos, California, 2004.

ISO/IEC - The International Organization For Standardization and the International Electrotechnial Commission (2008). "ISO/IEC 12207:2008 - Systems and software engineering - Software life cycle processes". Geneve: ISO.

Kitchenham, B. A. and Pfleeger, S. L. (2008). "Personal Opinion Surveys". Springer London, 2008. ISBN: 978-1-84800-044-5.

Murvey (2014). http://www.murvey.com. Último acesso em 20 de abril de 2014.

Pfleeger, S. L. and Kitchenham, B. A. (2001). "Principles of Survey Research Part 1 " Turning Lemons into Lemonade Systems / Software, I n c . What our series will discuss Description of the Lethbridge survey. v. 26, n. 6, p. 16-18. 
Pino, F. J., García, F. and Piattini, M. (2007). "Software process improvement in small and medium software enterprises: a systematic review". Software Quality Journal, v. 16 , n. 2, p. 237-261.

Runeson, P. and Höst, M. (2007) "Checklists for Software Engineering Case Study Research". In Proceedings of the First International Symposium on Empirical Software Engineering and Measurement, ESEM 2007. pp 479-481. Madrid, Spain, 2007.

SEI - SOFTWARE ENGINEERING INSTITUTE (2010). "Capability Maturity Model Integration for Development - CMMI-DEV 1.3". Version 1.3. Pittsburgh, PA. Carnegie Mellon University. USA, 2001.

SOFTEX - ASSOCIAÇÃO PARA PROMOÇÃO DA EXCELÊNCIA DO SOFTWARE BRASILEIRO (2012). "MPS . BR - Melhoria de Processo do Software Brasileiro Guia Geral MPS de Software". Disponível em http://www.softex.br. Brasil, 2012. 